\title{
Research Article \\ Self-Commutators of Composition Operators with Monomial Symbols on the Dirichlet Space
}

\author{
A. Abdollahi and S. Mehrangiz \\ Department of Mathematics, College of Sciences, Shiraz University, Shiraz 71454, Iran \\ Correspondence should be addressed to A. Abdollahi, abdollahi@shirazu.ac.ir
}

Received 14 June 2011; Accepted 10 August 2011

Academic Editor: Detlev Buchholz

Copyright (C 2011 A. Abdollahi and S. Mehrangiz. This is an open access article distributed under the Creative Commons Attribution License, which permits unrestricted use, distribution, and reproduction in any medium, provided the original work is properly cited.

Let $\varphi(z)=z^{n}, z \in \mathbb{U}$, for some positive integer $n$ and $C_{\varphi}$ the composition operator on the Dirichlet space $\Phi$ induced by $\varphi$. In this paper, we completely determine the point spectrum, spectrum, essential spectrum, and essential norm of the operators $C_{\varphi}^{*} C_{\varphi}, C_{\varphi} C_{\varphi}^{*}$ and self-commutators of $C_{\varphi}$, which expose that the spectrum and point spectrum coincide. We also find the eigenfunctions of the operators.

\section{Introduction}

Let $\varphi$ be a holomorphic self-map of the unit disk $\mathbb{U}:=\{z \in \mathbb{C}:|z|<1\}$. The function $\varphi$ induces the composition operator $C_{\varphi}$, defined on the space of holomorphic functions on $\mathbb{U}$ by $C_{\varphi} f=f \circ \varphi$. The restriction of $C_{\varphi}$ to various Banach spaces of holomorphic functions on $\mathbb{U}$ has been an active subject of research for more than three decades, and it will continue to be for decades to come (see [1-3]). Let $\Phi$ denote the Dirichlet space of analytic functions on the unit disk with derivatives that are square integrable with respect to the area measure on the disk. In recent years, the study of composition operators on the the Dirichlet space has received considerable attention (see [4-9] and references cited therein).

Let $\varphi(z)=z^{n}, z \in \mathbb{U}$, for some positive integer $n$, and $C_{\varphi}: \Phi \rightarrow \Phi$ the composition operator on the Dirichlet space $\Phi$ induced by $\varphi$. The main aim here is to find the spectrum, point spectrum, essential spectrum, and essential norm of $C_{\varphi}^{*} C_{\varphi}, C_{\varphi} C_{\varphi}^{*}$, self-commutator $\left[C_{\varphi}^{*}, C_{\varphi}\right]=C_{\varphi}^{*} C_{\varphi}-C_{\varphi} C_{\varphi}^{*}$ and anti-self-commutator $\left\{C_{\varphi}^{*}, C_{\varphi}\right\}=C_{\varphi}^{*} C_{\varphi}+C_{\varphi} C_{\varphi}^{*}$, for composition operators $C_{\varphi}$ on the Dirichlet space.

In [10], by using Cowen's formula for the adjoint of $C_{\varphi}$ on $H^{2}(\mathbb{U})$, the authors have completely determined the spectrum, essential spectrum, and point spectrum for selfcommutators of automorphic composition operators acting on the Hardy space of unit 
disk. In [4], the first author, has extended these results from the Hardy space to the Dirichlet space.

The other problem which is important to the study of composition operators is finding the relationships between the properties of the symbol $\varphi$ and essential normality of the composition operator $C_{\varphi}$. Recall that an operator $T$ on a Hilbert space $\mathscr{H}$ is called essentially normal if its image in the Calkin algebra is normal or equivalently if the self-commutator $\left[T^{*}, T\right]=$ $T^{*} T-T T^{*}$ is compact on $\mathscr{H}$.

In [11], the authors have determined which composition operators with automorphism symbol are essentially normal on $A^{2}\left(B_{N}\right)$ and $H^{2}\left(B_{N}\right)$ for $N \geq 1$. They have shown that the only essential normal automorphic composition operators are actually normal. This was first shown in the setting $H^{2}(\mathbb{U})$ by Zorboska in [12]. The related works and some historical remarks can be found in [10-13].

In [5], the authors consider composition operators $C_{\varphi}$, where $\varphi$ is a linear-fractional self-map of the unit disk $\mathbb{U}$, acting on the Dirichlet space $\boldsymbol{\Phi}$. By using the E. Gallardo and A. Montes' adjoint formula given in [6], they show that the essentially normal linear fractional composition operators on $\Phi$ are precisely those whose symbol is not a hyperbolic nonautomorphism with a boundary fixed point. They also obtained conditions for the linear fractional symbols $\varphi$ and $\psi$ of the unit disk for which $C_{\psi}^{*} C_{\varphi}$ or $C_{\varphi} C_{\psi}^{*}$ is compact.

In the next section, after giving some background material and presenting formula for the adjoint of $C_{\varphi}$ on $\Phi$, we give useful formula for the operators $C_{\varphi}^{*} C_{\varphi}, C_{\varphi} C_{\varphi}^{*},\left[C_{\varphi}^{*}, C_{\varphi}\right]$, and $\left\{C_{\varphi}^{*}, C_{\varphi}\right\}$, when $\varphi$ is an arbitrary monomial symbol $\varphi(z)=z^{n}$. In Section 3, we completely determine the point spectrum, spectrum, and essential spectrum of $C_{\varphi}^{*} C_{\varphi}$ and $C_{\varphi} C_{\varphi}^{*}$. Finally, in Section 4, we determine the same for $\left[C_{\varphi}^{*}, C_{\varphi}\right]$ and $\left\{C_{\varphi}^{*}, C_{\varphi}\right\}$.

\section{Preliminaries}

Throughout the paper, for a Hilbert space $\mathscr{H}, \mathbb{B}(\mathscr{L})$ denotes the set of bounded operators on $\mathscr{H}$ and $\boldsymbol{B}_{0}(\mathscr{H})$ denotes the closed ideal of all compact operators in $\mathcal{B}(\mathscr{H})$. The natural homomorphism of $\boldsymbol{B}(\mathscr{H})$ onto the quotient Banach algebra $\boldsymbol{B}(\mathscr{H}) / \boldsymbol{B}_{0}(\mathscr{H})=\mathbb{B} / \boldsymbol{B}_{0}$ - the Calkin algebra-is denoted by $T \mapsto \widetilde{T}=T+B_{0}(\mathscr{L})$.

For an operator $T \in B(\mathscr{\ell})$, the essential norm of $T$ is defined by

$$
\|T\|_{e}:=\inf \left\{\|T+K\|: K \in \mathfrak{B}_{0}(\mathscr{\ell})\right\}
$$

and the essential spectrum $\sigma_{\mathcal{e}}(T)$ is defined as the spectrum of the image $\tilde{T}$ of $T$ in the Calkin algebra $B(\mathscr{L}) / 乃_{0}(\mathscr{\ell})$. It is well known that the essential spectrum of a normal operator consists of all points in the spectrum of the operator except the isolated eigenvalues of finite multiplicity (see [14]).

As we mentioned in the Introduction, an operator $T$ on a Hilbert space $\mathscr{H}$ is called essentially normal if its image in the Calkin algebra is normal or equivalently if the self-commutator $\left[T^{*}, T\right]=T^{*} T-T T^{*}$ is compact on $\mathscr{d}$.

The Dirichlet space, which we denote by $\boldsymbol{\Phi}$, is the set of all analytic functions $f$ on the unit disk $\mathbb{U}$ for which

$$
\int_{\mathbb{U}}\left|f^{\prime}(z)\right|^{2} d A(z)<\infty
$$


where $d A$ denote the normalized area measure, and equivalently an analytic function $f$ is in $Ð$ if $\sum_{n=1}^{\infty} n|\widehat{f}(n)|^{2}<\infty$, where $\widehat{f}(n)$ denotes the $n$-th Taylor coefficient of $f$ at 0 . Background on the Dirichlet space can be found in [15] and the references cited therein.

For each holomorphic self-map $\varphi$ of $\mathbb{U}$, we define the composition operator $C_{\varphi}$ by $C_{\varphi} f=$ $f \circ \varphi(f \in \Phi)$.

Martín and Vukotić in [9] express and prove formulas for the adjoint of $C_{\varphi}$ on the Hardy space, when $\varphi$ is finite Blaschke product and also is rational self-map of the unit disk $\mathbb{U}$. By using the same arguments as in [9] for the Hardy space, one can prove the following theorem for the Dirichlet space case.

Theorem 2.1. Let $\varphi(z)=z^{n}$. For an arbitrary point $w=r e^{i \theta}$ in $\mathbb{U}$, writing its nth roots as $r_{k, w}=$ $r^{1 / n} e^{i(\theta+2 k \pi) / n}, k=0,1, \ldots, n-1$. The adjoint of $C_{\varphi}$ (viewed as an operator on the Dirichlet space $\boldsymbol{\oplus}$ ) is given by the formula

$$
C_{\varphi}^{*} f(w)=\sum_{k=0}^{n-1} f\left(r_{k, w}\right)-(n-1) f(0)
$$

Throughout this paper, we denote by $\mathcal{M}$ the closed subspace of $\Phi$ spanned by the monomials $\left\{z^{n k}: k=0,1, \ldots\right\}$ and by $P_{\mathcal{M}}: \Phi \rightarrow \mathcal{M}$ the corresponding orthogonal projection onto $\mathcal{M}$.

Remark 2.2. Let $\varphi(z)=z^{n}$ and $r_{k, \varphi(w)}=r^{1 / n} e^{i(\theta+2 k \pi) / n}, k=0,1, \ldots, n-1$, be the $n$th roots of $\varphi(z)=\varphi(w)$. For $f \in \Phi$ with $f(z)=\sum_{m=0}^{\infty} c_{m} z^{m}$, we have

$$
\begin{aligned}
\sum_{k=0}^{n-1} f\left(r_{k, \varphi(w)}\right) & =\sum_{k=0}^{n-1} \sum_{m=0}^{\infty} c_{m} r_{k, \varphi(w)}^{m} \\
& =\sum_{m=0}^{\infty} c_{m} \sum_{k=0}^{n-1} r_{k, \varphi(w)}^{m} \\
& =n \sum_{j=0}^{\infty} c_{n j} w^{n j}=n\left(P_{\mathcal{M}} f\right)(w) .
\end{aligned}
$$

Before stating our main results, we also need the next results.

Theorem 2.3. Let $\varphi(z)=z^{n}$. Then,

$$
\begin{gathered}
C_{\varphi}^{*} C_{\varphi}=n I \bmod B_{0}(\boldsymbol{\Phi}) \\
\left(C_{\varphi} C_{\varphi}^{*} f\right)(w)=n\left(P_{\mathcal{M}} f\right)(w)-(n-1) f(0),
\end{gathered}
$$

$\boldsymbol{B}_{0}(\boldsymbol{\Phi})$ is the ideal of compact operators on $\boldsymbol{\Phi}$. 
Proof. By a simple computation and using formula (2.3), it follows that

$$
\begin{gathered}
\left(C_{\varphi}^{*} C_{\varphi} f\right)(w)=n f(w)-(n-1) f(0) \\
\left(C_{\varphi} C_{\varphi}^{*} f\right)(w)=\sum_{k=0}^{n-1} f\left(r_{k, \varphi(w)}\right)-(n-1) f(0),
\end{gathered}
$$

for each $w \in \mathbb{U}, f \in \Phi$. Thus,

$$
\begin{gathered}
C_{\varphi}^{*} C_{\varphi}=n I \bmod \boldsymbol{B}_{0}(\boldsymbol{\Phi}) \\
\left(C_{\varphi} C_{\varphi}^{*} f\right)(w)=n\left(P_{\mathcal{M}} f\right)(w)-(n-1) f(0) .
\end{gathered}
$$

\section{Spectrum of $C_{\varphi}^{*} C_{\varphi}$ and $C_{\varphi} C_{\varphi}^{*}$}

Let $\varphi(z)=z^{n}$. In this section, we are going to find the point spectrum, spectrum, essential spectrum, and the eigenfunctions of the operators $C_{\varphi}^{*} C_{\varphi}$ and $C_{\varphi} C_{\varphi}^{*}$.

Theorem 3.1. Let $\varphi(z)=z^{n}$. Then,

$$
\sigma_{e}\left(C_{\varphi}^{*} C_{\varphi}\right)=\{n\}, \quad \sigma_{p}\left(C_{\varphi}^{*} C_{\varphi}\right)=\sigma\left(C_{\varphi}^{*} C_{\varphi}\right)=\{1, n\},
$$

and, for $n \geq 2$,

$$
\sigma_{e}\left(C_{\varphi} C_{\varphi}^{*}\right)=\{0, n\}, \quad \sigma_{p}\left(C_{\varphi} C_{\varphi}^{*}\right)=\sigma\left(C_{\varphi} C_{\varphi}^{*}\right)=\{1,0, n\},
$$

and, in the case that $n=1$,

$$
\sigma_{p}\left(C_{\varphi} C_{\varphi}^{*}\right)=\sigma_{e}\left(C_{\varphi} C_{\varphi}^{*}\right)=\sigma\left(C_{\varphi} C_{\varphi}^{*}\right)=\{1\}
$$

Proof. Since the operator $C_{\varphi}^{*} C_{\varphi}$ is a finite rank perturbation of $n I$, the essential spectrum of this operator is $\{n\}$. Since any points in the spectrum of a normal operator which are not in the essential spectrum are isolated eigenvalues of finite multiplicity, it is enough to find the eigenvalues. We first do this for the operator $C_{\varphi}^{*} C_{\varphi}$. Let $\lambda \in \mathbb{C}$ be an eigenvalue of the operator $C_{\varphi}^{*} C_{\varphi}$ with corresponding eigenvector $f \in \Phi$. Then, $C_{\varphi}^{*} C_{\varphi} f=\lambda f$. By using formula (2.7) for $C_{\varphi}^{*} C_{\varphi}$, we have

$$
n f(w)-(n-1) f(0)=\lambda f(w), \quad w \in \mathbb{U}
$$

By putting $w=0$, it follows that $n f(0)-(n-1) f(0)=\lambda f(0)$. If $f(0) \neq 0$, then $\lambda=1$. Thus for the case $\lambda=1$, the function $f(w)=f(0)$ is a nonzero function in $\Phi$ that satisfies the equation, and, hence, $\lambda=1$ is an eigenvalue of the operator $C_{\varphi}^{*} C_{\varphi}$. If $f(0)=0$, then $\lambda=n$, and, in this 
case, the function $w^{k}, k \geq 1$ is a nonzero function in $\Phi$ that satisfies (3.4). Hence, $\lambda=n$ is an eigenvalue of the operator $C_{\varphi}^{*} C_{\varphi}$ with infinite multiplicity. So

$$
\sigma_{p}\left(C_{\varphi}^{*} C_{\varphi}\right)=\sigma\left(C_{\varphi}^{*} C_{\varphi}\right)=\{1, n\}
$$

Now, let $\lambda \in \mathbb{C}$ be an eigenvalue of the operator $C_{\varphi} C_{\varphi}^{*}$ with corresponding eigenvector $f \in \boldsymbol{\Phi}$. Then, $C_{\varphi} C_{\varphi}^{*} f=\lambda f$. By using formula (2.6) for $C_{\varphi} C_{\varphi}^{*}$, we have

$$
n\left(P_{\mathcal{M}} f\right)(w)-(n-1) f(0)=\lambda f(w)
$$

By putting $w=0$, it follows that

$$
n f(0)-(n-1) f(0)=\lambda f(0) .
$$

If $f(0) \neq 0$, then $\lambda=1$. Thus,

$$
n\left(P_{\mathcal{M}} f\right)(w)-(n-1) f(0)=f(w) .
$$

Let $f(z)=\sum_{m=0}^{\infty} c_{m} z^{m}$. Then,

$$
n \sum_{j=0}^{\infty} c_{n j} w^{n j}-(n-1) c_{0}=\sum_{m=0}^{\infty} c_{m} w^{m}, \quad w \in \mathbb{U}
$$

For $n \geq 2$, it follows that $c_{m}=0$, whenever $m \geq 1$. So $f \equiv 1$ is an eigenfunction corresponding to $\lambda=1$. Thus, $\lambda=1$ is an eigenvalue of the operator $C_{\varphi} C_{\varphi}^{*}$.

Now suppose that $f(0)=0$. Then, $n P_{\mathcal{M}} f=\lambda f$ and so $\lambda / n$ is an eigenvalue of $P_{\mathcal{M}}$. Hence, $\lambda=0$ or $\lambda=n$.

So, for the case $\lambda=0$ and $n \geq 2$, the function $w$ is a nonzero function in $\Phi$ that satisfies (3.6), and, hence, $\lambda=0$ is an eigenvalue of the operator $C_{\varphi} C_{\varphi}^{*}$.

So when $n \geq 2$, the eigenvalues of $C_{\varphi} C_{\varphi}^{*}$ are $\{1,0, n\}$. In the case that $\lambda=n$, for each natural number $k, w^{n k}$ is a nonzero function in $\Phi$ that satisfies (3.6), and, hence, the essential spectrum $C_{\varphi} C_{\varphi}^{*}$ contains $n$. If $\lambda=0$ and $n \geq 2$, then we conclude that for each natural number $m$ which is not a multiple of $n, w^{m}$ is a nonzero function in $\Phi$ that satisfies (3.6), and, hence, $\lambda=0$ is an eigenvalue of the operator $C_{\varphi} C_{\varphi}^{*}$ with infinite multiplicity. So the essential spectrum $C_{\varphi} C_{\varphi}^{*}$ contains 0 . Since

$$
\sigma\left(C_{\varphi} C_{\varphi}^{*}+\mathbb{B}_{0}(\boldsymbol{\Phi})\right) \cup\{0\}=\sigma\left(C_{\varphi}^{*} C_{\varphi}+\boldsymbol{B}_{0}(\boldsymbol{\Phi})\right) \cup\{0\}
$$

and $\sigma_{e}\left(C_{\varphi}^{*} C_{\varphi}\right)=\{n\}$, for $n \geq 2$, we conclude that

$$
\sigma_{e}\left(C_{\varphi} C_{\varphi}^{*}\right)=\{0, n\}
$$


So when $\varphi(z)=z^{n}$ and $n \geq 2$,

$$
\sigma_{p}\left(C_{\varphi} C_{\varphi}^{*}\right)=\sigma\left(C_{\varphi} C_{\varphi}^{*}\right)=\{1,0, n\}
$$

and, for $n=1$,

$$
\sigma_{p}\left(C_{\varphi} C_{\varphi}^{*}\right)=\sigma_{e}\left(C_{\varphi} C_{\varphi}^{*}\right)=\sigma\left(C_{\varphi} C_{\varphi}^{*}\right)=\{1\}
$$

\section{The Spectrum of $\left[C_{\varphi^{\prime}}^{*} C_{\varphi}\right]$ and $\left\{C_{\varphi^{\prime}}^{*} C_{\varphi}\right\}$}

Theorem 4.1. Let $\varphi(z)=z^{n}$. Then, for $n=1$,

$$
\sigma_{p}\left(\left[C_{\varphi}^{*}, C_{\varphi}\right]\right)=\sigma_{e}\left(\left[C_{\varphi}^{*}, C_{\varphi}\right]\right)=\sigma\left(\left[C_{\varphi}^{*}, C_{\varphi}\right]\right)=\{0\}
$$

and, for $n \geq 2$,

$$
\sigma_{p}\left(\left[C_{\varphi}^{*}, C_{\varphi}\right]\right)=\sigma_{e}\left(\left[C_{\varphi}^{*}, C_{\varphi}\right]\right)=\sigma\left(\left[C_{\varphi}^{*}, C_{\varphi}\right]\right)=\{0, n\}
$$

Proof. Let $T=C_{\varphi}^{*} C_{\varphi}-C_{\varphi} C_{\varphi}^{*}$. Then,

$$
T=n\left(I-P_{\mathcal{M}}\right)
$$

Since any points in the spectrum of a normal operator which are not in the essential spectrum are isolated eigenvalues of finite multiplicity, we first find the eigenvalues.

If $n \geq 2$, then $I-P_{\mathscr{M}}$ is a nontrivial projection and so $\sigma(T)=\{0, n\}$.

In the case that $\lambda=n$, for each natural number $m$ which is not a multiple of $n$, the function $w^{m}$ is an eigenfunction of $T$, and, hence, $\lambda=n$ is an eigenvalue of the operator $T$ with infinite multiplicity. For the case $\lambda=0$, for each natural number $k, w^{k n}$ is an eigenfunction of $T$, and, hence, $\lambda=0$ is an eigenvalue of the operator $T$ with infinite multiplicity.

The essential spectrum of $\left[C_{\varphi}^{*}, C_{\varphi}\right]$ can be computed directly by using the following:

$$
\begin{aligned}
& \sigma_{e}\left(\left[C_{\varphi}^{*}, C_{\varphi}\right]\right)=\sigma\left(C_{\varphi}^{*} C_{\varphi}-C_{\varphi} C_{\varphi}^{*}+B_{0}(刃)\right) \\
& =\sigma\left(n I-C_{\varphi} C_{\varphi}^{*}+B_{0}(\Phi)\right) .
\end{aligned}
$$

So if $\varphi(z)=z$, then

$$
\sigma_{p}\left(\left[C_{\varphi}^{*}, C_{\varphi}\right]\right)=\sigma_{e}\left(\left[C_{\varphi}^{*}, C_{\varphi}\right]\right)=\sigma\left(\left[C_{\varphi}^{*}, C_{\varphi}\right]\right)=\{0\}
$$


and, if $\varphi(z)=z^{n}$ and $n \geq 2$, then

$$
\sigma_{p}\left(\left[C_{\varphi}^{*}, C_{\varphi}\right]\right)=\sigma_{e}\left(\left[C_{\varphi}^{*}, C_{\varphi}\right]\right)=\sigma\left(\left[C_{\varphi}^{*}, C_{\varphi}\right]\right)=\{0, n\}
$$

Theorem 4.2. Let $\varphi(z)=z^{n}$. Then, for $n=1$,

$$
\sigma_{p}\left(\left\{C_{\varphi}^{*}, C_{\varphi}\right\}\right)=\sigma_{e}\left(\left\{C_{\varphi}^{*}, C_{\varphi}\right\}\right)=\sigma\left(\left\{C_{\varphi}^{*}, C_{\varphi}\right\}\right)=\{2\}
$$

for $n \geq 2$,

$$
\begin{gathered}
\sigma_{e}\left(\left\{C_{\varphi}^{*}, C_{\varphi}\right\}\right)=\{2 n, n\}, \\
\sigma_{p}\left(\left\{C_{\varphi}^{*}, C_{\varphi}\right\}\right)=\sigma\left(\left\{C_{\varphi}^{*}, C_{\varphi}\right\}\right)=\{2, n, 2 n\} .
\end{gathered}
$$

Proof. Let $S=C_{\varphi}^{*} C_{\varphi}+C_{\varphi} C_{\varphi}^{*}$. Then for each $f \in \Phi$ and $w \in \mathbb{U}$,

$$
(S f)(w)=n f(w)+n\left(P_{\mathcal{M}} f\right)(w)-2(n-1) f(0) .
$$

Since $S$ is self-adjoint, any points in the spectrum of $S$ which are not in the essential spectrum are eigenvalues of finite multiplicity. So we first find such points.

Let $\lambda \in \mathbb{C}$ be an eigenvalue of the operator $S$ with corresponding eigenvector $f \in \mathbb{D}$. Then, $S f=\lambda f$. So we have

$$
n f(w)+n\left(P_{\mathcal{M}} f\right)(w)-2(n-1) f(0)=\lambda f(w) .
$$

By putting $w=0$, it follows that $2 n f(0)-2 n f(0)+2 f(0)=\lambda f(0)$. If $f(0) \neq 0$, then $\lambda=2$. Thus,

$$
n f(w)+n\left(P_{\mathcal{M}} f\right)(w)-2(n-1) f(0)=2 f(w) .
$$

The function $f(w)=1$ is a nonzero function in $\Phi$ that satisfies the equation, and, hence, $\lambda=2$ is an eigenvalue of the operator $S$. If $f(0)=0$, then

$$
n\left(P_{\mathcal{M}} f\right)(w)=(\lambda-n) f(w),
$$

and it follows that $\lambda=n$ or $\lambda=2 n$. For the case $\lambda=n$ with $n \geq 2$, for each natural number $m$ which is not a multiple of $n$, the function $w^{m}$ is a nonzero function in $\Phi$ that satisfies (4.12), and, hence, $\lambda=n$ is an eigenvalue of the operator $S$ with infinite multiplicity. In the case that $\lambda=2 n$, for each natural number $k, w^{k n}$ is a nonzero function in $\Phi$ that satisfies (4.12), 
and, hence, $\lambda=2 n$ is an eigenvalues of the operator $S$ with infinite multiplicity. The essential spectrum of $\left\{C_{\varphi}^{*}, C_{\varphi}\right\}$ can be computed directly by using the following:

$$
\begin{aligned}
& \sigma_{e}\left(\left\{C_{\varphi}^{*}, C_{\varphi}\right\}\right)=\sigma\left(C_{\varphi}^{*} C_{\varphi}+C_{\varphi} C_{\varphi}^{*}+B_{0}(\boldsymbol{\nexists})\right) \\
& =\sigma\left(n I+C_{\varphi} C_{\varphi}^{*}+B_{0}(\boldsymbol{\Phi})\right) .
\end{aligned}
$$

Hence, we conclude that when $\varphi(z)=z^{n}$ and $n \geq 2$,

$$
\sigma_{e}\left(\left\{C_{\varphi}^{*}, C_{\varphi}\right\}\right)=\{n, 2 n\}
$$

Also, if $\varphi(z)=z$, then

$$
\sigma_{p}\left(\left\{C_{\varphi}^{*}, C_{\varphi}\right\}\right)=\sigma_{e}\left(\left\{C_{\varphi}^{*}, C_{\varphi}\right\}\right)=\sigma\left(\left\{C_{\varphi}^{*}, C_{\varphi}\right\}\right)=\{2\}
$$

and, for $n \geq 2$,

$$
\sigma_{p}\left(\left\{C_{\varphi}^{*}, C_{\varphi}\right\}\right)=\sigma\left(\left\{C_{\varphi}^{*}, C_{\varphi}\right\}\right)=\{2, n, 2 n\}
$$

\section{Acknowledgments}

The authors would like to thank the editor of the Abstract and Applied Analysis and the referee for useful and helpful comments and suggestions.

\section{References}

[1] J. H. Shapiro, Composition Operators and Classical Function Theory, Springer, New York, NY, USA, 1993.

[2] R. K. Singh and J. S. Manhas, Composition Operators on Function Spaces, vol. 179, North-Holland, Amsterdam, The Netherlands, 1993.

[3] C. C. Cowen and B. D. MacCluer, Composition Operators on Spaces of Analytic Functions, CRC Press, Boca Raton, Fla, USA, 1995.

[4] A. Abdollahi, "Self-commutators of automorphic composition operators on the Dirichlet space," Proceedings of the American Mathematical Society, vol. 136, no. 9, pp. 3185-3193, 2008.

[5] G. A. Chacón and G. R. Chacón, "Some properties of composition operators on the Dirichlet space," Acta Mathematica Universitatis Comenianae, vol. 74, no. 2, pp. 259-272, 2005.

[6] E. A. Gallardo-Gutiérrez and A. Montes-Rodríguez, "Adjoints of linear fractional composition operators on the Dirichlet space," Mathematische Annalen, vol. 327, no. 1, pp. 117-134, 2003.

[7] C. Hammond, "The norm of a composition operator with linear symbol acting on the Dirichlet space," Journal of Mathematical Analysis and Applications, vol. 303, no. 2, pp. 499-508, 2005.

[8] M. J. Martín and D. Vukotić, "Norms and spectral radii of composition operators acting on the Dirichlet space," Journal of Mathematical Analysis and Applications, vol. 304, no. 1, pp. 22-32, 2005.

[9] M. J. Martín and D. Vukotić, "Adjoints of composition operators on Hilbert spaces of analytic functions," Journal of Functional Analysis, vol. 238, no. 1, pp. 298-312, 2006.

[10] P. S. Bourdon and B. D. MacCluer, "Self-commutators of automorphic composition operators," Complex Variables and Elliptic Equations, vol. 52, no. 1, pp. 85-104, 2007. 
[11] B. D. MacCluer and M. A. Pons, "Automorphic composition operators on Hardy and Bergman spaces in the ball," Houston Journal of Mathematics, vol. 32, no. 4, pp. 1121-1132, 2006.

[12] N. Zorboska, "Closed range essentially normal composition operators are normal," Acta Scientiarum Mathematicarum, vol. 65, no. 1-2, pp. 287-292, 1999.

[13] B. D. MacCluer and R. J. Weir, "Essentially normal composition operators on Bergman spaces," Acta Scientiarum Mathematicarum, vol. 70, no. 3-4, pp. 799-817, 2004.

[14] J. B. Conway, A Course in Functional Analysis, vol. 96, Springer, New York, NY, USA, 2nd edition, 1990.

[15] W. T. Ross, "The classical Dirichlet space," in Recent Advances in Operator-Related Function Theory, vol. 393 of Contemporary Mathematics, pp. 171-197, American Mathematical Society, Providence, RI, USA, 2006. 


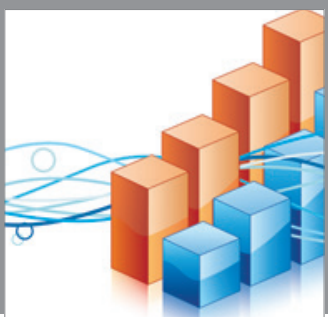

Advances in

Operations Research

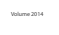

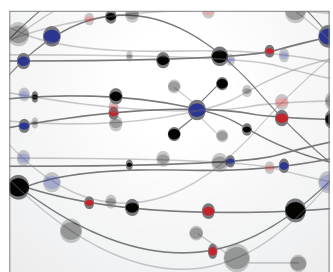

\section{The Scientific} World Journal
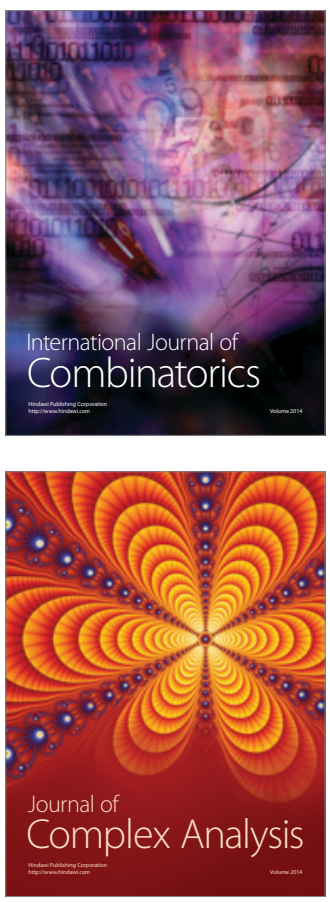

International Journal of

Mathematics and

Mathematical

Sciences
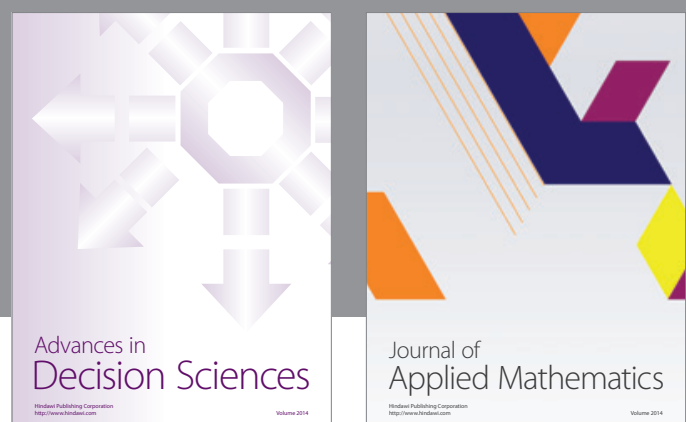

Journal of

Applied Mathematics
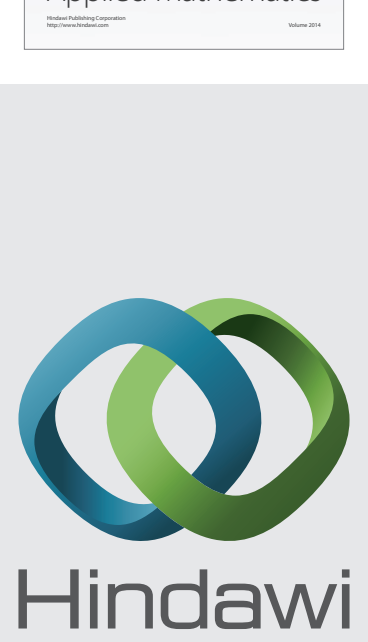

Submit your manuscripts at http://www.hindawi.com
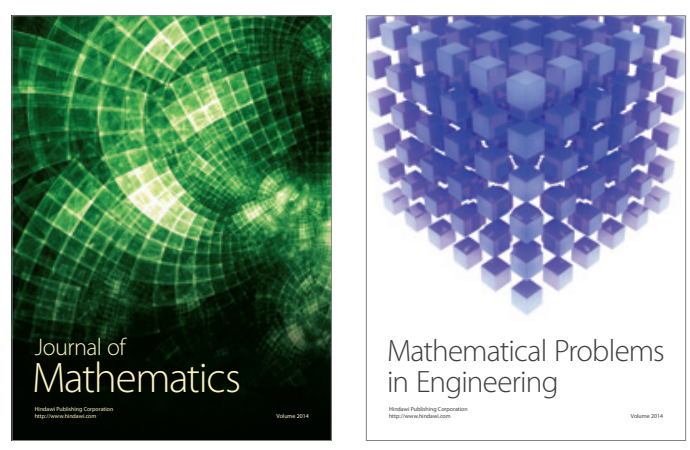

Mathematical Problems in Engineering
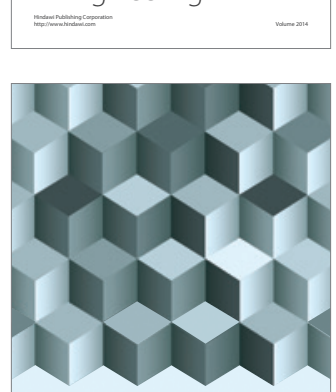

Journal of

Function Spaces
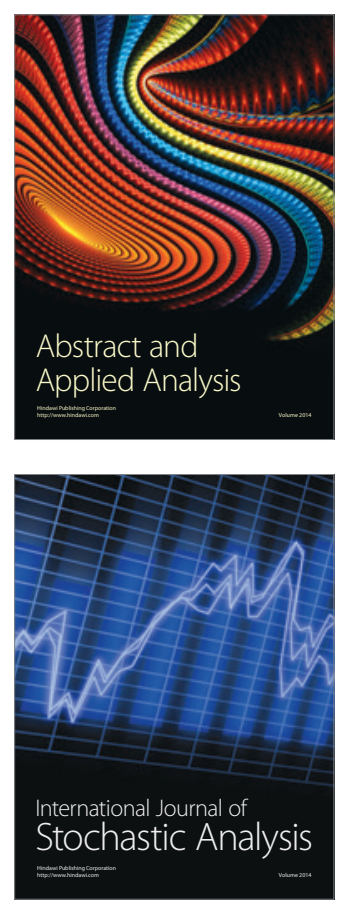

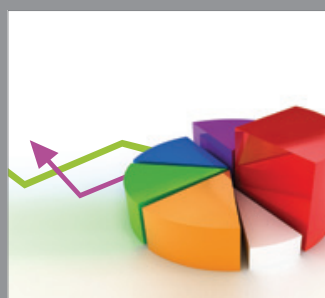

ournal of

Probability and Statistics

Promensencen
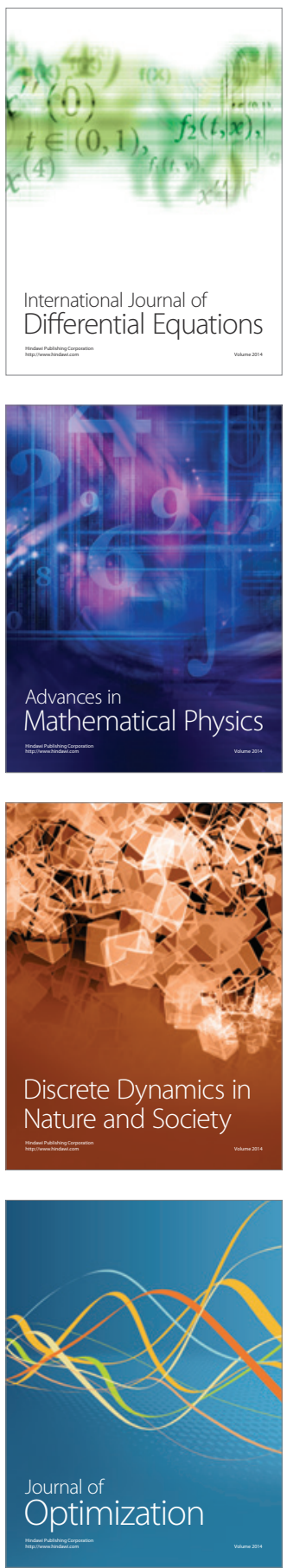